\title{
Share Desktop Screen to Raspberry Pi
}

\author{
Tarun Jain \\ Student of Masters of Technology (Computer Science and Engineering) ASET, Amity University Noida, Uttar \\ Pradesh, India
}

Abstract: Our aim is to connect laptops to projectors/TV/LED's wirelessly (without any physical media) and transfer video signals from them. For this, SSH connection is used in Raspberry Pi device and we have transferred command using CGI.

Index Terms- Raspberry Pi, WIFI, VNC, SSH.

\section{Introduction}

Raspberry $\mathrm{P}^{[1]}$ is a credit card sized microcomputer capable of performing all necessary functions performed by any computer. This innovative device is developed by Newark element14 (Premier Farnell), RS Components and Egoman.

Raspberry Pi comes in two models; Model-A and Model-B. The main difference between them is their RAM capacity. Rest all hardware is approximately same. It has Broadcom BCM 2835 system on a chip (SOC), video core GPU (Graphical processing unit) and ARM1176JZF-S $700 \mathrm{MHz}$ processor (which can attempt overclocking up to $1 \mathrm{GHz}$ with the help of "Turbo" mode). Rest hardware includes SD card slot (SD card as a memory for storing OS and data), HDMI Port, RCA video port, 3mm audio jack, two USB 2.0 jacks, RJ45 Ethernet and micro USB power.

\section{Idea}

Our idea is to connect raspberry pi with projectors and connect laptops with raspberry pi device using $\mathrm{WIFI}^{[2]}$. After connecting, we can give command to raspberry pi to start VNC viewer by the window application installed in our laptops (customized application developed in .net). This window application will start $\mathrm{VNC}^{[3]}$ server in laptops and open the CGI window of raspberry. With a single click on application VNC viewer command will execute in raspberry pi automatically. Hence, this is what required. We are able to transfer our laptop screens to raspberry pi, which is eventually connected to projectors.

\section{Working}

I have installed "Raspbian" OS to raspberry pi using NOOBS on 8 GB SD card. T-Link WIFI USB adapter is connected to raspberry pi device and a WIFI connection is made. Keyboard and mouse are required for the first time. After successful connection, I was able to connect my raspberry using SSH connection. That's all done! Now, I have installed VNC viewer using command: sudo apt-get install vnc viewer.

Then apache server is installed with $\mathrm{PHP}^{[4]}$. Now, my raspberry is ready to work as the server. I could see my home page by typing IP address of raspberry pi in the browser window of any laptop, desktop.

On laptop, VNC server is installed and we can create a window application that can run VNC server in laptop and send IP of laptop to raspberry pi using CGI and run a python script that will start VNC viewer in raspberry pi.

\section{Acknowledgment}

I would like to thank Dr. Sanjeev Bansal (Director of Amity Business School, Noida, India) for his continuous support in my work and guiding me the way to achieve my goal. I would also like to thank Prof. Sanjeev Thakur (Professor, Amity School of Engineering and Technology, Noida, India) for helping in technical topics.

\section{References}

\footnotetext{
[1] http://en.wikipedia.org/wiki/Raspberry Pi

[2] https://learn.adafruit.com/adafruits-raspberry-pi-lesson-3-network-setup/setting-up-wifi-with-occidentalis

[3] http://myraspberrypiexperience.blogspot.com/p/setting-up-vnc.html

[4] http://www.penguintutor.com/linux/light-webserver
} 